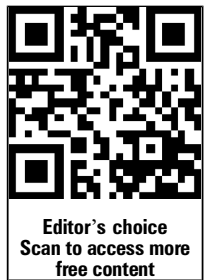

free content

\title{
Cardiovascular risk stratification in rheumatic diseases: carotid ultrasound is more sensitive than Coronary Artery Calcification Score to detect subclinical atherosclerosis in patients with rheumatoid arthritis
}

\author{
Alfonso Corrales, ${ }^{1}$ José A Parra, ${ }^{2}$ Carlos González-Juanatey, ${ }^{3}$ Javier Rueda-Gotor, ${ }^{1}$ \\ Ricardo Blanco, ${ }^{1}$ Javier Llorca, ${ }^{4}$ Miguel A González-Gay ${ }^{1}$
}

Handling editor Tore K Kvien

- Additional material is published online only. To view please visit the journal online (http://dx.doi.org/10.1136/ annrheumdis-2013-203688).

For numbered affiliations see end of article.

\section{Correspondence to} Dr Miguel A González-Gay, Rheumatology Division, Hospital Universitario Marqués de Valdecilla, IFIMAV, Avenida de Valdecilla, s/n, 39008, Santander, Cantabria 39008, Spain; miguelaggay@hotmail. com

AC, JAP and CG-J contributed equally to this work and share first authorship.

$J$ and MAG-G shared senior authorship in this study.

Received 26 March 2013 Revised 12 May 2013 Accepted 26 June 2013 Published Online First 13 July 2013

\section{SLinked}

http://dx.doi.org/10.1136/ annrheumdis-2013-203682

- http://dx.doi.org/10.1136/ annrheumdis-2013-203911

To cite: Corrales $A$, Parra JA, González Juanatey $C$, et al. Ann Rheum Dis 2013:72. 1764-1770.

\section{ABSTRACT}

Objective To determine the ability of Coronary Artery Calcification Score (CACS) and carotid ultrasonography in detecting subclinical atherosclerosis in rheumatoid arthritis (RA).

Methods A set of 104 consecutive RA patients without history of cardiovascular (CV) events were studied to determine CACS, carotid intima-media thickness (CIMT) and plaques. Systematic Coronary Risk Evaluation (SCORE) modified according to the EULAR recommendations (mSCORE) was also assessed. Results The mean disease duration was 10.8 years, $72.1 \%$ had rheumatoid factor and/or anti-CCP positivity and $16.4 \%$ extra-articular manifestations. Nine were excluded because they had type 2 diabetes mellitus or chronic kidney disease. CV risk was categorised in the remaining 95 RA patients according to the mSCORE as follows: low $(n=21)$, moderate $(n=60)$ and high/very high risk $(n=14)$. Most patients with low mSCORE (16/21; 76.2\%) had normal CACS (zero), and none of them CACS $>100$. However, a high number of patients with carotid plaques was disclosed in the groups with CACS $0(23 / 40 ; 57.5 \%)$ or CACS 1-100 (29/38: $76.3 \%) .72(75.8 \%)$ of the 95 patients fulfilled definitions for high/very high CV as they had an mSCORE $\geq 5 \%$ or $\mathrm{mSCORE}<5 \%$ plus one of the following findings: severe carotid ultrasonography findings (cIMT>0.9 mm and/or plaques) or CACS $>100$. A CACS $>100$ showed sensitivity similar to mSCORE (23.6\% vs $19.4 \%)$. In contrast, the presence of severe carotid ultrasonography findings allowed identifying most patients who met definitions for high/very high CV risk (70/72; sensitivity $97.2 \%$ (95\% Cl 90.3 to 99.7)).

Conclusions Carotid ultrasonography is more sensitive than CACS for the detection of subclinical atherosclerosis in $R A$.

\section{INTRODUCTION}

Rheumatoid arthritis (RA) is the prototype of disease associated with accelerated atherosclerosis and increased rate of cardiovascular (CV) disease. ${ }^{12}$ The mechanisms leading to an elevated $\mathrm{CV}$ mortality rate in RA are complex, including traditional $\mathrm{CV}$ and the presence of other factors such as a chronic inflammatory state and a genetic compound. ${ }^{3-6}$ Therefore, a comprehensive assessment and treatment of traditional and nontraditional $\mathrm{CV}$ risk factors should form part of the routine care of the patient with RA. ${ }^{7}$ However, adequate stratification of the $\mathrm{CV}$ risk in patients with RA is still far from being completely established. Classic risk assessment tools used to estimate the $\mathrm{CV}$ risk in the general population may not fully predict the development of future CV events in patients with RA. Reports showing patients who did not reach values to be considered as having high $\mathrm{CV}$ risk according to these $\mathrm{CV}$ risk estimates, such as the Systematic Coronary Risk Evaluation (SCORE) modified according to the European League Against Rheumatism (EULAR) recommendations, ${ }^{8}$ who suffered $\mathrm{CV}$ complications, mainly ischaemic heart disease, have been reported. ${ }^{9}$ Therefore, the search for additional tools that may help to identify high-risk patients, who may benefit from active therapy to prevent CV events, is needed. It may be of major importance in RA patients who are not included in the categories of high or very high CV risk according to the classic risk assessment tools.

Several validated non-invasive imaging techniques offer a unique opportunity to study the relation of surrogate markers to the development of atherosclerosis. ${ }^{10}$ They may be useful to determine subclinical atherosclerosis in RA patients. Among them, the assessment of carotid intima-media thickness (cIMT) and the presence of plaques by carotid ultrasonography has become an affordable, efficient technique to measure the presence of subclinical atherosclerosis in RA. ${ }^{10} 11$ Both cIMT and carotid plaques were found to be good predictors of $\mathrm{CV}$ events in low and intermediate risk groups of nonrheumatic individuals and also in patients with RA. ${ }^{12-14}$ We previously proposed that carotid ultrasonography assessment should be considered in the $\mathrm{CV}$ risk assessment of patients with RA who do not fulfil definitions for high $\mathrm{CV}$ risk according to the SCORE. ${ }^{15}$ In keeping with this proposal, a recent study has confirmed that carotid ultrasonography assessment may be useful to establish the actual CV risk in RA, in particular in patients with moderate SCORE. ${ }^{16}$

A question that needs to be answered is whether other non-invasive techniques may also be comparable with carotid ultrasonography to stratify the $\mathrm{CV}$ risk of patients with RA. Among them, coronary artery calcification (CAC) has proved to be a predictor of $\mathrm{CV}$ events in the general population. ${ }^{17}$ 
In this regard, Coronary Artery Calcification Score (CACS) assessed by the multi-detector CT (MDCT) scan was reported to be useful in the assessment of the extension and severity of atherosclerosis in vascular beds. Using this technique, an increased prevalence of CAC has been observed in patients with RA. ${ }^{18}$ A recent study has shown that CAC detected by the MDCT is a good predictor of CV events in patients with RA. ${ }^{19}$

Taking together all these considerations, in the present study we aimed to determine the ability of CACS and carotid ultrasonography to detect subclinical atherosclerosis in RA. We also sought to establish the relationship between carotid ultrasonography findings and CACS and whether the use of CACS may improve the stratification of the CV risk of RA patients without clinically evident CV disease.

\section{PATIENTS AND METHODS \\ Patients}

A set of 104 consecutive patients with a diagnosis of RA recruited from Hospital Universitario Marqués de Valdecilla (Santander, Northern Spain) who were seen over a 3-month period were included in the present study. All the patients who were assessed for MDCT and carotid ultrasonography fulfilled the 1987 American College of Rheumatology classification criteria and the 2010 classification criteria for RA. ${ }^{20-22}$ Patients with a history of CV events (ischaemic heart disease, cerebrovascular accident, peripheral arterial disease or heart failure that were defined as previously reported) were not recruited in this study. $^{5} 13$ Then, clinical records of all patients were again reviewed in an attempt to fully establish comorbidities. Patients with type 1 diabetes with target organ damage, type 2 diabetes mellitus or those who had severe chronic kidney disease are considered as having very high $\mathrm{CV}$ risk according to current guidelines and they were excluded from the final analysis. ${ }^{5} 23$ Patients were considered as having extra-articular manifestations as previously reported. ${ }^{5}$

\section{SCORE risk estimation}

Based on a pool of datasets from 12 European cohort studies, mainly carried out in general population settings, European experts performed the SCORE project to develop a risk scoring system for use in the clinical management of CV risk in the European clinical practice. ${ }^{23}$ The SCORE risk estimation system offers direct estimation of fatal $\mathrm{CV}$ risk in a format suited to the constraints of clinical practice. ${ }^{24}$ It estimates the 10 -year risk of a first fatal atherosclerotic event, whether heart attack, stroke or other occlusive arterial disease, including sudden cardiac death. Risk estimates have been produced as charts for high- and low-risk regions in Europe. ${ }^{23}$ Spain was included in the low-risk region in Europe.

Both SCORE and EULAR modified SCORE (mSCORE) according to the EULAR recommendations were calculated to determine the 10 -year risk of fatal $\mathrm{CV}$ disease in a population at a low CV disease risk. ${ }^{8}{ }^{23}$ SCORE chart assessment was based on the following risk factors: age, gender, smoking, systolic blood pressure and atherogenic index (total cholesterol/highdensity lipoprotein-cholesterol). ${ }^{8} \quad 23$ The subject's written consent was obtained in all the cases. The study was approved by the local Ethical Committee.

\section{MDCT imaging assessment and analysis of data}

To determine CACS, all subjects underwent CT imaging of coronary arteries using a 32-slice multi-detector computed tomography (MDCT) scanner (Lightspeed, Pro 32, GE Healthcare, USA). It was performed following current guidelines on the screening for CAC for cardiac risk assessment. ${ }^{25}$ Patient's score was calculated as the sum of calcium score in the left main coronary artery, left anterior descending artery, left circumflex coronary artery, right coronary artery and posterior descending artery. Patients were further stratified into four groups according to their range of CACS: $\mathrm{CACS}=0, \mathrm{CACS}=1-100, \mathrm{CACS}=101-$ 400 and CACS $>400$.

In general, the higher the score the more likely the patient is going to have coronary heart disease. CACS $>100$ indicates a high probability of coronary artery disease. Based on CACS results, a CACS 0 is normal; CACS $1-10$ indicates low CV risk; CACS 11-100 moderate CV risk; CACS 101-400 moderate to high $\mathrm{CV}$ risk; and $\mathrm{CAC}>400$ high $\mathrm{CV}$ risk. ${ }^{26}$ For the purpose of estimation of high/very high $\mathrm{CV}$ risk we used as cut-off a CACS $>100$.

\section{Carotid ultrasonography examination}

Carotid ultrasonography examination included the measurement of cIMT in the common carotid artery and the detection of focal plaques in the extracranial carotid tree. ${ }^{16} 27$ Additional information on Methods is provided in the online supplementary material.

Like in the general population, cIMT $>0.90$ or the presence of plaques are predictors of CV events in RA. ${ }^{13}{ }^{14}$ Therefore, patients having cIMT $>0.90$ or plaques were included in the category of high/very high CV risk.

\section{Statistical analysis}

Results were expressed as number (percentage) or mean $\pm \mathrm{SD}$. Equality of percentages was tested using the Fisher exact test (two-tailed). To estimate the sensitivity to establish the presence of high/very high $\mathrm{CV}$ risk we considered the presence of $\mathrm{mSCORE} \geq 5 \%$ or $\mathrm{mSCORE} \geq 1 \%$ and $<5 \%$ plus one of the following imaging findings: severe carotid ultrasonography findings (cIMT $>0.9 \mathrm{~mm}$ and/or carotid plaques) or CACS $>100 ; 95 \%$ CI for sensitivity were estimated assuming a binomial distribution. Goodman-Kruskal $\gamma$ test was used to measure the strength of association and correlation of the cross-tabulated data on CACS and the variables measured at the ordinal levels. This test was used to determine correlation between CACS and cIMT and between CACS and carotid plaques. A p value $<0.05$ was considered statistically significant. All the statistical tests were performed with the package Stata V.12/SE (Stata Corp, College Station, Texas, USA).

\section{RESULTS}

Most patients from this series of 104 RA patients without CV events were women $(73 ; 70.2 \%)$. The age at the time of the study and disease duration (mean \pm SD) was $59.3 \pm 10.3$ and $10.8 \pm 8.3$ years, respectively. Rheumatoid factor and/or anticyclic citrullinated peptide (anti-CCP) positivity was found in $75(72.1 \%)$ cases and $17(16.4 \%)$ had extra-articular manifestations. The SCORE (mean \pm SD) was $2.30 \pm 2.53$ and the EULAR mSCORE $2.78 \pm 3.28$. A cIMT $>0.90 \mathrm{~mm}$ was observed in $17(16.4 \%)$ patients and carotid plaques in $77(74.0 \%)$; 16 of the 17 patients with cIMT $>0.90$ also had carotid plaques. Other characteristics of this series are shown in table 1.

\section{SCORE risk, EULAR mSCORE risk, CACS and carotid ultrasonography results}

RA patients were stratified according to the SCORE analysis (table 2). When the risk model was adapted using the multiplication factor to establish the EULAR mSCORE, only $4(5.7 \%)$ of the 70 patients initially categorised as having moderate CV 
Table 1 Main features of a series of 104 patients with rheumatoid arthritis without cardiovascular events

\begin{tabular}{|c|c|c|}
\hline Variable & Category & Number (\%) \\
\hline Sex & Women & $73(70.2)$ \\
\hline Rheumatoid factor & Positive & $61(58.7)$ \\
\hline Anti-CCP & Positive & $60(57.7)$ \\
\hline Rheumatoid factor and/or anti-CCP & At least one of them positive & $75(72.1)$ \\
\hline Presence of extra-articular manifestations* & Yes & $17(16.4)$ \\
\hline Smoking & Yes & $42(40.4)$ \\
\hline Carotid intima-media thickness $>0.90 \mathrm{~mm}$ & Yes & $17(16.4)$ \\
\hline Carotid plaques & Yes & $77(74.0)$ \\
\hline CACS $>100$ & Yes & $21(20.2)$ \\
\hline Variable & & Mean (SD) \\
\hline Age at the time of the carotid ultrasonography assessment (SD), years & & $59.3(10.3)$ \\
\hline Disease duration (SD), years & & $10.8(8.3)$ \\
\hline Systolic blood pressure (SD), mm Hg & & $135.6(19.9)$ \\
\hline Diastolic blood pressure (SD), $\mathrm{mm} \mathrm{Hg}$ & & $78.9(8.1)$ \\
\hline Total cholesterol (SD), mg/dL & & $215.2(43.6)$ \\
\hline HDL-cholesterol (SD), mg/dL & & $63.4(19.2)$ \\
\hline Atherogenic index (SD) & & $3.62(1.11)$ \\
\hline SCORE† (SD) & & $2.30(2.53)$ \\
\hline Modified SCORE $¥$ (SD) & & $2.78(3.28)$ \\
\hline Carotid intima-media thickness (SD), $\mathrm{mm}$ & & $0.75(0.16)$ \\
\hline
\end{tabular}

risk $(\geq 1 \%$ and $<5 \%$ ) fulfilled definitions for high and/or very high $\mathrm{CV}$ risk (EULAR $\mathrm{mSCORE} \geq 5 \%$ ). The frequency of normal MDCT results, manifested by a CACS 0 , was higher in patients with low mSCORE $(<1 \%)$ (16 of $21 ; 76.2 \%)$ than in patients with moderate/high/very high mSCORE (25 of $83 ; 30.1 \%$ ); $\mathrm{OR}=7.4$ (95\% CI 2.2 to $28.2 ; \mathrm{p}=0.0001$ ).

None of the patients from this series had type 1 diabetes with target organ damage. However, nine fulfilled definitions for type 2 diabetes mellitus or had severe chronic kidney disease and were excluded from further analyses. Because of this, SCORE, mSCORE, carotid ultrasonography and CACS data were reassessed in the remaining 95 cases (tables 3 and 4). Following this procedure, only $3(4.8 \%)$ of the 63 patients categorised as having moderate $\mathrm{CV}$ risk according to the SCORE were reclassified as having high or very high CV risk when the EULAR mSCORE was applied.

A good correlation between the mSCORE and the CACS score was found (Goodman-Kruskal $\gamma$ test $=0.6104 ; \mathrm{p}<0.001$ ). In this regard, most patients with a low mSCORE (16/21 $(76.2 \%)$ ) had normal score (CACS 0 ), and none of these patients with a low mSCORE had a CACS $>100$. Also, most patients with a high or very high mSCORE (12/14 (85.7\%)) had an abnormal score (CACS $\geq 1$ ). However, 38 of 60 patients $(63.3 \%)$ with a moderate mSCORE had a CACS $\geq 1$ and 12 (20\%) had a CACS $>100$. Therefore, although a low mSCORE was associated with a normal CACS, it was not the case for

Table 2 Cardiovascular risk stratification according to SCORE risk* and EULAR modified SCORE riskt in 104 rheumatoid arthritis patients without cardiovascular events

\begin{tabular}{|c|c|c|c|c|c|c|}
\hline & \multirow[b]{3}{*}{$\begin{array}{l}\text { SCORE* } \\
\text { n }\end{array}$} & \multicolumn{5}{|c|}{ EULAR modified SCORE† } \\
\hline & & \multirow[b]{2}{*}{$\mathbf{n}$} & \multicolumn{4}{|l|}{ CACS } \\
\hline & & & $\begin{array}{l}\text { Normal } \neq(\text { CACS 0) } \\
\mathrm{n}=41(\%)\end{array}$ & $\begin{array}{l}\text { Low-moderate } \\
\text { (CACS 1-100) } \\
n=42(\%)\end{array}$ & $\begin{array}{l}\text { Moderate-high } \\
\text { (CACS >100-400) } \\
n=12(\%)\end{array}$ & $\begin{array}{l}\text { High (CACS >400) } \\
n=9(\%)\end{array}$ \\
\hline Low (<1\%) & 21 & 21 & 16/21 (76.2.0) & 5/21 (23.8) & $0 / 21(0.0)$ & $0 / 21(0.0)$ \\
\hline Moderate $(\geq 1 \%$ and $<5 \%)$ & 70 & 66 & 22/66 (33.3) & $28 / 66(42.4)$ & 9/66 (13.6) & $7 / 66(10.6)$ \\
\hline High ( $\geq 5 \%$ and $<10 \%)$ & 11 & 13 & 2/13 (15.4) & 7/13 (53.8) & $3 / 13(23.1)$ & $1 / 13(7.7)$ \\
\hline Very high $(\geq 10 \%)$ & 2 & 4 & $1 / 4(25.0)$ & $2 / 4(50.0)$ & $0 / 4(0.0)$ & $1 / 4(25.0)$ \\
\hline High plus very high & 13 & 17 & 3/17 (17.7) & 9/17 (52.9) & 3/17 (17.7) & 2/17 (11.7) \\
\hline
\end{tabular}

EULAR modified SCORE risk stratification according to the Coronary Artery Calcification Score (CACS).

*According to SCORE for the general population 2324

TModified SCORE for rheumatoid arthritis: according to the EULAR recommendations. ${ }^{8}$

¥Modified SCORE was calculated by the application of a multiplier factor of 1.5 in those patients with two of three criteria: disease duration $>10$ years, rheumatoid factor and/or anticyclic citrullinated peptide antibody positivity, and presence of extra-articular manifestations. ${ }^{8}$

EULAR, European League Against Rheumatism; SCORE, Systematic Coronary Risk Evaluation. 
Table 3 Cardiovascular risk stratification according to SCORE risk* and EULAR modified SCORE riskt in 95 rheumatoid arthritis patients without cardiovascular events, diabetes mellitus or severe chronic kidney diseaseł

\begin{tabular}{|c|c|c|c|c|c|c|}
\hline \multirow[b]{3}{*}{ Cardiovascular risk } & \multirow[b]{3}{*}{$\begin{array}{l}\text { SCORE* } \\
\mathrm{n}\end{array}$} & \multicolumn{5}{|c|}{ EULAR modified SCORE§ } \\
\hline & & \multirow[b]{2}{*}{$\mathbf{n}$} & \multicolumn{4}{|l|}{ CACS } \\
\hline & & & $\begin{array}{l}\text { Normal‡ (CACS } 0) \\
\mathrm{n}=40(\%)\end{array}$ & $\begin{array}{l}\text { Low-moderate } \\
\text { (CACS 1-100) } \\
n=38(\%)\end{array}$ & $\begin{array}{l}\text { Moderate-high } \\
\text { (CACS >100-400) } \\
\mathrm{n}=9(\%)\end{array}$ & $\begin{array}{l}\text { High } \\
\text { (CACS >400) } \\
n=8(\%)\end{array}$ \\
\hline Low $(<1 \%)$ & 21 & 21 & $16 / 21(76.2)$ & $5 / 21(23.8)$ & $0 / 21(0.0)$ & $0 / 21(0.0)$ \\
\hline Moderate $(\geq 1 \%$ and $<5 \%)$ & 63 & 60 & $22 / 60(36.7)$ & $26 / 60(43.3)$ & $6 / 60(10.0)$ & $6 / 60(10.0)$ \\
\hline High $(\geq 5 \%$ and $<10 \%)$ & 9 & 10 & $1 / 10(10.0)$ & $5 / 10(50.0)$ & $3 / 10(30.0)$ & $1 / 10(10.0)$ \\
\hline Very high $(\geq 10 \%)$ & 2 & 4 & $1 / 4(25.0)$ & $2 / 4(50.0)$ & $0 / 4(0.0)$ & $1 / 4(25.0)$ \\
\hline High plus very high & 11 & 14 & 2/14 (14.3) & $7 / 14(50.0)$ & $3 / 14(21.4)$ & 2/14 (14.3) \\
\hline \multicolumn{7}{|c|}{$\begin{array}{l}\text { EULAR modified SCORE risk stratification according to the Coronary Artery Calcification Score (CACS). } \\
{ }^{*} \text { According to SCORE for the general population. } 23.24 \\
\text { tModified SCORE for rheumatoid arthritis: according to the EULAR recommendations. }{ }^{8} \\
\text { \#Six patients with moderate modified SCORE and three with high and/or very high modified SCORE had type } 2 \text { diabetes or severe chronic kidney disease and, due to this, met } \\
\text { definitions for very high cardiovascular risk and were excluded from the analysis. } \\
\text { §Modified SCORE was calculated by the application of a multiplier factor of } 1.5 \text { in those patients with two of three criteria: disease duration }>10 \text { years, rheumatoid factor and/or } \\
\text { anticyclic citrullinated peptide antibody positivity, and presence of extra-articular manifestations. } \\
\text { EULAR, European League Against Rheumatism; SCORE, Systematic Coronary Risk Evaluation. }\end{array}$} \\
\hline
\end{tabular}

moderate mSCORE. Of note, table 4 showed a high frequency $(85.0 \%)$ of severe carotid ultrasonography abnormalities in patients with a moderate mSCORE.

\section{Correlation between CACS and the cIMT}

A cIMT $>0.90 \mathrm{~mm}$ was the cut-off associated with increased risk of $\mathrm{CV}$ events in the general population and in patients with RA. ${ }^{13}$ Most patients with $\mathrm{cIMT} \leq 0.9 \mathrm{~mm}$ had a normal CACS (Agatston 0) (table 5). The frequency of cIMT $>0.9 \mathrm{~mm}$ was increased in patients with abnormal CACS $(\geq 1)$. Therefore, a significant correlation between cIMT and CACS was found $(\mathrm{p}=0.02)$.

\section{Correlation between CACS and the presence of carotid plaques}

Table 6 shows the presence of carotid plaques in RA stratified according to CACS. Significant correlation between CACS and presence of carotid plaques was observed (Goodman-Kruskal $\gamma$ test $=0.6375 ; \mathrm{p}<0.001)$. The correlation remained statistically significant when patients were stratified according to the presence of unilateral or bilateral carotid plaques (table 7). Most patients with $C A C S \geq 1$ had carotid plaques (46/55 (83.6\%)). It was especially true for those with a CACS $>100$ as all of them (17/17) exhibited carotid plaques. In addition, the majority of patient with a CACS $>100$ had bilateral plaques that indicated the presence of severe atherosclerotic disease.

However, a high number of RA patients with carotid plaques was disclosed in the group of patients with a CACS $=0(23 / 40$ $(57.5 \%))$. Therefore, the use of carotid ultrasonography allowed classifying more than $50 \%$ of patients as having very high CV risk who would have not been included in this category if the only tool for classification had been CACS results. It was also the case for a CACS $1-100$ as $29 / 38$ (76.3\%) patients with a CACS 1-100 had carotid plaques.

\section{Model to establish the presence of high/very high CV risk in patients with RA and moderate mSCORE}

We aimed to establish whether CACS assessed by MTCD and carotid ultrasonography may enhance the identification of high/ very high $\mathrm{CV}$ risk in patients with RA and moderate EULAR mSCORE. For this purpose, we defined high/very high CV risk regardless of $\mathrm{mSCORE}$ results if patients had severe ultrasonography findings (cIMT $>0.90 \mathrm{~mm}$ and/or plaques) or a CACS $>100$.

Table 4 Cardiovascular risk stratification according to SCORE risk* and EULAR modified SCORE riskt in 95 rheumatoid arthritis patients without cardiovascular events, diabetes mellitus or severe chronic kidney disease

\begin{tabular}{|c|c|c|c|c|c|c|c|}
\hline \multirow[b]{3}{*}{ Cardiovascular risk } & \multirow[b]{3}{*}{$\begin{array}{l}\text { SCORE* } \\
n\end{array}$} & \multicolumn{6}{|c|}{ EULAR modified SCORE $\ddagger$} \\
\hline & & \multirow[b]{2}{*}{$\mathbf{n}$} & \multirow{2}{*}{$\begin{array}{l}\text { CACS } \\
\begin{array}{l}\text { CACS }>100 \\
n=17(\%)\end{array}\end{array}$} & \multicolumn{4}{|c|}{ Carotid ultrasonography } \\
\hline & & & & $\begin{array}{l}\text { CIMT>0.90 mm } \\
\mathrm{n}=14(\%)\end{array}$ & $\begin{array}{l}\text { Carotid plaques } \\
\mathrm{n}=69(\%)\end{array}$ & $\begin{array}{l}\text { cIMT }>0.90 \mathrm{~mm} \text { and/or } \\
\text { carotid plaques } \\
\mathrm{n}=70(\%)\end{array}$ & $\begin{array}{l}\text { cIMT } \leq 0.90 \mathrm{~mm} \text { and } \\
\text { without carotid plaques } \\
\mathrm{n}=25(\%)\end{array}$ \\
\hline Low $(<1 \%)$ & 21 & 21 & $0 / 21(0.0)$ & $0 / 21(0.0)$ & $7 / 21(33.3)$ & $7 / 21(33.3)$ & $14 / 21(66.7)$ \\
\hline Moderate $(\geq 1 \%$ and $<5 \%)$ & 63 & 60 & $12 / 60(20.0)$ & $8 / 60(13.3)$ & $51 / 60(85.0)$ & $51 / 60(85.0)$ & 9/60 (15.0) \\
\hline High $(\geq 5 \%$ and $<10 \%)$ & 9 & 10 & $4 / 10(40.0)$ & $5 / 10(50.0)$ & $8 / 10(80.0)$ & $9 / 10(90.0)$ & $1 / 10(10.0)$ \\
\hline Very high $(\geq 10 \%)$ & 2 & 4 & $1 / 4(25.0)$ & $1 / 4(25.0)$ & $3 / 4(75.0)$ & $3 / 4(75.0)$ & $1 / 4(25.0)$ \\
\hline High plus very high & 11 & 14 & $5 / 14(35.7)$ & $6 / 14(42.8)$ & $11 / 14(78.6)$ & $12 / 14(85.7)$ & $2 / 14(14.3)$ \\
\hline
\end{tabular}

EULAR modified SCORE risk stratification according to the Coronary Artery Calcification Score (CACS) $>100$, carotid intima-media thickness (cIMT) $>0.90$ mm and carotid plaques. *According to SCORE for the general population ${ }^{23} 24$

tModified SCORE for rheumatoid arthritis: according to the EULAR recommendations. ${ }^{8}$

¥Modified SCORE was calculated by the application of a multiplier factor of 1.5 in those patients with two of three criteria: disease duration $>10$ years, rheumatoid factor and/or anticyclic citrullinated peptide antibody positivity, and presence of extra-articular manifestations. ${ }^{8}$

EULAR, European League Against Rheumatism; SCORE, Systematic Coronary Risk Evaluation. 
Table 5 Correlation between the Coronary Artery Calcification Score (CACS) and the carotid intima-media thickness (cIMT) in 95 patients with rheumatoid arthritis

\begin{tabular}{|c|c|c|}
\hline \multirow[b]{2}{*}{ CACS } & \multicolumn{2}{|l|}{ cIMT } \\
\hline & $\begin{array}{l}\leq 0.90 \mathrm{~mm} \\
\mathrm{n}(\%)\end{array}$ & $\begin{array}{l}>0.90 \mathrm{~mm} \\
\mathrm{n}(\%)\end{array}$ \\
\hline 0 & $37 / 40(92.5)$ & $3 / 40(7.5)$ \\
\hline $1-100$ & $32 / 38(84.2)$ & $6 / 38(15.8)$ \\
\hline $101-400$ & $5 / 9(55.6)$ & $4 / 9(44.4)$ \\
\hline$>400$ & $7 / 8(88.8)$ & $1 / 8(12.5)$ \\
\hline$\geq 1$ & $44 / 55(80.0)$ & $11 / 55(20.0)$ \\
\hline
\end{tabular}

In all, 60 RA patients fulfilled definitions for moderate $\mathrm{CV}$ mSCORE ( $\geq 1 \%$ and $<5 \%$ ) (tables 3 and 4 ). Overall, 51 of these 60 patients were reclassified as having a high or very high mSCORE risk because of the presence of severe ultrasonography findings (cIMT $>0.9 \mathrm{~mm}$ and/or carotid plaques) or CACS $>100$. Interestingly, carotid ultrasonography allowed reclassifying as having high/very high $\mathrm{CV}$ risk all these 51 patients while using only a CAC $>100$ as the criterion for high/ very high CV risk we only reclassified 12 of these 51 patients.

Taking together these results, in patients with a moderate mSCORE $(\geq 1 \%$ and $<5 \%)$, the sensitivity for high/very high $\mathrm{CV}$ risk using carotid ultrasonography definitions of severity (cIMT $>0.90 \mathrm{~mm}$ and/or plaques) was $100 \%$. In contrast, the sensitivity shown by CACS assessed by MDCT using CACS $>100$, a criterion of high/very high CV risk, was $23.5 \%$. Therefore, CACS seems to be inferior to carotid ultrasonography to detect high/very high CV risk in patients with moderate mSCORE.

\section{Sensitivity to establish the presence of high/very high CV risk in patients with RA using $\mathrm{mSCORE}$, carotid ultrasonography or MDCT}

A total of 72 of the 95 patients without diabetes mellitus or severe chronic kidney disease fulfilled definitions for high/very high $\mathrm{CV}$ risk: a) $\mathrm{mSCORE} \geq 5 \%$ or $\mathrm{b}) \mathrm{mSCORE}<5 \%$ plus one of the following: severe carotid ultrasonography findings (cIMT $>0.9 \mathrm{~mm}$ and/or plaques) or CACS $>100$ (table 8). A CACS $>100$ was found to have sensitivity similar to that observed using only mSCORE charts $(23.6 \%$ and $19.4 \%$, respectively). A slight increase in the sensitivity was observed when an mSCORE $>5 \%$ plus the presence of a CACS $>100$ in patients with an mSCORE $<5 \%$ was used $(36.1 \%$ (95\% CI 25.2 to 48.3$)$ ). Interestingly, the presence of severe ultrasonography

\begin{tabular}{|c|c|c|}
\hline \multirow[b]{2}{*}{ CACS } & \multicolumn{2}{|c|}{ Carotid plaques } \\
\hline & $\begin{array}{l}\text { No } \\
\text { n (\%) }\end{array}$ & $\begin{array}{l}\text { Yes } \\
\text { n (\%) }\end{array}$ \\
\hline 0 & $17 / 40(42.5)$ & $23 / 40(57.5)$ \\
\hline $1-100$ & 9/38 (23.7) & $29 / 38(76.3)$ \\
\hline $101-400$ & 0/9 (0.0) & 9/9 (100.0) \\
\hline$>400$ & $0 / 8(0.0)$ & $8 / 8(100.0)$ \\
\hline$\geq 1$ & 9/55 (16.4) & 46/55 (83.6) \\
\hline
\end{tabular}

Table 7 Correlation between the Coronary Artery Calcification Score (CACS) and the presence of unilateral or bilateral carotid plaques in 95 patients with rheumatoid arthritis

\begin{tabular}{llll}
\hline \multirow{4}{*}{ CACS } & \multicolumn{2}{c}{ Carotid plaques } & \\
\cline { 2 - 4 } & No & Unilateral & Bilateral \\
\hline 0 & 17 & 12 & 11 \\
$1-100$ & 9 & 14 & 15 \\
$101-400$ & 0 & 1 & 8 \\
$>400$ & 0 & 1 & 7 \\
\hline Goodman-Kruskal $\gamma$ test $=0.5644 ; p<0.001$. &
\end{tabular}

findings allowed identifying most patients categorised as having high/very high CV risk (70 of 72; sensitivity $97.2 \%$ (95\% CI 90.3 to 99.7$)$ ). In addition, the use of mSCORE $>5 \%$ plus an $\mathrm{mSCORE}<5 \%$ and the presence of severe carotid ultrasonography results (cIMT $>0.9 \mathrm{~mm}$ and/or carotid plaques) allowed identifying all the 72 patients (sensitivity 100\% (95\% CI 95.0 to 100$)$ ).

\section{DISCUSSION}

Adequate stratification of the $\mathrm{CV}$ risk is an issue of major importance in the management of patients with RA. CACS determined by MDCT scanner and carotid ultrasonography are non-invasive tools to measure atherosclerosis that have been recommended as possible additions to risk factor assessment charts for predicting the probability of occurrence of CV events in non-rheumatic patients. In the present study we assessed for first time a comparative relation of CACS and carotid ultrasonography results after integration into the EULAR mSCORE in patients with RA.

Unlike carotid ultrasonography, the mSCORE does not significantly improve the identification of RA patients with high CV risk. ${ }^{16}$ In the present study, we observed good correlation

Table 8 Sensitivity to establish the presence of high/very high cardiovascular risk in rheumatoid arthritis patients without cardiovascular events, diabetes mellitus or severe chronic kidney disease using the modified EULAR SCORE, severe carotid ultrasound findings (cIMT>0.90 mm or carotid plaques) or a Coronary Artery Calcification Score (CACS) $>100$

\begin{tabular}{ll}
\hline Model & Sensitivity \\
\hline Gold standard* & $\mathrm{n}=72 / 95$ \\
Modified SCORE $>5 \%$ & $\mathrm{n}=14$ of 72 \\
& $19.4 \%$ \\
& $(95 \% \mathrm{Cl} 11.1$ to 30.5$)$ \\
CACS $>100$ & $\mathrm{n}=17$ of 72 \\
& $23.6 \%$ \\
& $(95 \% \mathrm{Cl} 14.4$ to 35.1$)$ \\
cIMT $>0.90 \mathrm{~mm}$ & $\mathrm{n}=70$ of 72 \\
and/or & $97.2 \%$ \\
carotid plaques & $(95 \% \mathrm{Cl} 90.3$ to 99.7$)$ \\
Modified SCORE $>5 \%$ & $\mathrm{n}=26$ of 72 \\
or & $36.1 \%$ \\
modified SCORE $<5 \%$ plus CACS $>100$ & $(95 \% \mathrm{Cl} 25.2$ to 48.3$)$ \\
Modified SCORE $>5 \%$ & $\mathrm{n}=72$ of 72 \\
or & $100 \%$ \\
modified SCORE $<5 \%$ plus one of the following: & $(95 \%$ Cl 95.0 to 100$)$ \\
clMT $>0.90$ mm or carotid plaques & \\
\hline *Gold standard for high/very high cardiovascular risk: & \\
Modified SCORE $\geq 5 \%$ OR Modified SCORE $<5 \%$ plus one of the following: severe \\
carotid ultrasound findings (clMT>0.90 mm or carotid plaques) or a CACS $>100$. \\
EULAR, European League Against Rheumatism; SCORE, Systematic Coronary Risk \\
Evaluation.
\end{tabular}


between the mSCORE and the CACS. We also observed good correlation between CACS and cIMT as only a few patients from our series with CACS 0 had cIMT>0.90 mm while the frequency of cIMT $>0.90$ was higher among patients with CACS $\geq 1$. However, several reports indicate that in the general population measurements of carotid ultrasonography and CACS are often discordant. ${ }^{28} 29$ In keeping with these observations, we disclosed that a substantial number of RA patients without CV disease, who apparently did not have atherosclerosis according to CACS results, had severe carotid ultrasonography abnormalities, mainly carotid plaques. Carotid ultrasonography disclosed the presence of plaques in a high number of RA patients with CACS 0 or CACS $1-100$. This finding is of particular relevance as the presence of severe carotid ultrasonography findings predicts CV events in RA. ${ }^{13} 14$

The high number of cases with severe ultrasonography findings among patients with moderate mSCORE might raise doubts on the possibility that some of them would be wrongly overtreated. Nevertheless, previous studies have confirmed that a cIMT $>0.90 \mathrm{~mm}$ is indicative of atherosclerosis and increased risk of $\mathrm{CV}$ disease $(0.90 \mathrm{~mm}$ was found to be a useful cut-off value to predict the presence of coronary artery disease). ${ }^{30}$ Carotid plaques are also predictors of coronary disease progression and closely associated with coronary artery disease. ${ }^{31}$

In assessing the correlation between CACS and carotid plaques, we found that the frequency of carotid plaques was higher in RA patients with CACS $>100$ than in those with CACS 0 or $1-100$. However, only $20 \%$ of patients with moderate mSCORE had CACS $>100$ while $85 \%$ of them exhibited carotid plaques in the ultrasonography evaluation. These findings indicate that in patients with RA carotid ultrasonography may be a more sensitive test than CACS for the detection of subclinical atherosclerosis. These results support the use of carotid ultrasonography as the imaging technique of choice for detection of high/very high CV risk in RA patients with moderate mSCORE. The value of CACS is probably limited for this group of patients. In this regard, although arterial calcification indicates a later stage of vascular disease, its absence does not exclude the presence of non-calcified 'vulnerable' plaques. In keeping with our observations, in a series of 118 young to middle-aged, low-risk population who reported 1 or more CV risk factors and were seeking primary prevention but who did not have symptoms of CV disease, a high frequency of severe carotid ultrasonography findings in individuals with CACS 0 was observed. $^{32}$ Similarly, in another series of 136 asymptomatic individuals without $\mathrm{CV}$ disease, severe carotid ultrasonography findings defined by cIMT $\geq 75$ percentile and/or plaques were more likely to revise Framingham risk score than CACS. ${ }^{33}$ In this series, $52 \%$ of the subjects with CACS 0 had plaques. ${ }^{33}$ This percentage was remarkably similar to that observed in our series as we found carotid plaques in $57.5 \%$ of RA patients with CACS 0.

In conclusion, the use of an mSCORE does not allow identifying properly high/very high CV risk RA patients. Carotid ultrasonography assessment can detect subclinical vascular disease in RA with $\mathrm{CACS} \leq 100$. Therefore, carotid ultrasonography seems to be more sensitive than CACS in the stratification of the CV risk of patients with RA.

\footnotetext{
Author affiliations

'Division of Rheumatology, Hospital Universitario Marqués de Valdecilla, Santander, Cantabria, Spain

2Division of Radiology, Hospital Universitario Marqués de Valdecilla, Santander, Cantabria, Spain

${ }^{3}$ Division of Cardiology, Hospital Lucus Augusti, Lugo, Cantabria, Spain
}

${ }^{4}$ Division of Epidemiology and Computational Biology, School of Medicine, University of Cantabria, Santander, and CIBER Epidemiología y Salud Pública (CIBERESP), Santander, Cantabria, Spain

Contributors AC performed the carotid ultrasound study, contributed to the elaboration of the protocol of study, helped in the interpretation of data and in the elaboration of the manuscript. JAP performed the multi-detector CT study, contributed to the elaboration of the protocol of study, helped in the interpretation of data and in the elaboration of the manuscript. CG-J contributed to the elaboration of the protocol of study, helped in the interpretation of data and the elaboration of the manuscript. JR-G contributed to the elaboration of the manuscript. RB recruited patients for the study and contributed to the elaboration of the manuscript. $J L$ contributed to the elaboration of the protocol of study, helped in the interpretation of data and the elaboration of the manuscript and performed the statistical analysis. MAG-G recruited patients for the study, contributed to the elaboration of the protocol of study, helped in the interpretation of data and was responsible of the final drafting and elaboration of the manuscript.

Funding This study was supported by grants from 'Fondo de Investigaciones Sanitarias' PI06/0024, PS09/00748 and PI12/00060 (Spain). This work was also partially supported by RETICS Program, RD08/0075 and RD12/0009/0013 (RIER) from 'Instituto de Salud Carlos III' (ISCIII) (Spain).

Competing interests None.

Patient consent Obtained.

Ethics approval Cantabrian (Spain) Ethical Committee.

Provenance and peer review Not commissioned; externally peer reviewed.

\section{REFERENCES}

1 Gonzalez-Gay MA, Gonzalez-Juanatey C, Martin J. Rheumatoid arthritis: a disease associated with accelerated atherogenesis. Semin Arthritis Rheum 2005;35:8-17.

2 Aviña-Zubieta JA, Choi HK, Sadatsafavi M, et al. Risk of cardiovascular mortality in patients with rheumatoid arthritis: a meta-analysis of observational studies. Arthritis Rheum 2008;59:1690-7.

3 Dessein $\mathrm{PH}$, Joffe Bl, Veller MG, et al. Traditional and nontraditional cardiovascular risk factors are associated with atherosclerosis in rheumatoid arthritis. I Rheumatol 2005:32:435-42.

4 Del Rincon ID, Williams K, Stern MP, et al. High incidence of cardiovascular events in rheumatoid arthritis cohort not explained by traditional cardiac risk factors. Arthritis Rheum 2001;44:2737-45.

5 Gonzalez-Gay MA, Gonzalez-Juanatey C, Lopez-Diaz MJ, et al. HLA-DRB1 and persistent chronic inflammation contribute to cardiovascular events and cardiovascular mortality in patients with rheumatoid arthritis. Arthritis Rheum 2007;57:125-32.

6 Rodríguez-Rodríguez L, González-Juanatey C, Palomino-Morales R, et al. TNFA-308 (rs1800629) polymorphism is associated with a higher risk of cardiovascular disease in patients with rheumatoid arthritis. Atherosclerosis 2011;216:125-30.

7 Dessein $\mathrm{PH}$, Joffe BI. When is a patient with rheumatoid arthritis at risk for cardiovascular disease? J Rheumatol 2006;33:201-3.

8 Peters MJ, Symmons DP, McCarey D, et al. EULAR evidence-based recommendations for cardiovascular risk management in patients with rheumatoid arthritis and other forms of inflammatory arthritis. Ann Rheum Dis 2010;69:325-31.

9 Crowson CS, Gabriel SE. Towards improving cardiovascular risk management in patients with rheumatoid arthritis: the need for accurate risk assessment. Ann Rheum Dis 2011;70:719-21.

10 Kerekes G, Soltész P, Nurmohamed MT, et al. Validated methods for assessment of subclinical atherosclerosis in rheumatology. Nat Rev Rheumatol 2012;8:224-34.

11 Gonzalez-Gay MA, Gonzalez-Juanatey C, Vazquez-Rodriguez TR, et al. Endothelial dysfunction, carotid intima-media thickness, and accelerated atherosclerosis in rheumatoid arthritis. Semin Arthritis Rheum 2008;38:67-70.

12 Nambi V, Chambless L, Folsom AR, et al. Carotid intima-media thickness and presence or absence of plaque improves prediction of coronary heart disease risk: the ARIC (Atherosclerosis Risk In Communities) study. J Am Coll Cardiol 2010;55:1600-7

13 Gonzalez-Juanatey C, Llorca J, Martin J, et al. Carotid intima-media thickness predicts the development of cardiovascular events in patients with rheumatoid arthritis. Semin Arthritis Rheum 2009;38:366-71.

14 Evans MR, Escalante A, Battafarano DF, et al. Carotid atherosclerosis predicts incident acute coronary syndromes in rheumatoid arthritis. Arthritis Rheum 2011;63:1211-20.

15 González-Gay MA, González-Juanatey C, Llorca J. Carotid ultrasound in the cardiovascular risk stratification of patients with rheumatoid arthritis: when and for whom? Ann Rheum Dis 2012;71:796-8.

16 Corrales A, González-Juanatey C, Peiró ME, et al. Carotid ultrasound is useful for the cardiovascular risk stratification of patients with rheumatoid arthritis: results of a population-based study. Ann Rheum Dis 2013. Published Online First: 16 Mar 2013. doi:10.1136/annrheumdis-2013-203101 
17 Detrano R, Guerci AD, Carr JJ, et al. Coronary calcium as a predictor of coronary events in four racial or ethnic groups. N Engl J Med 2008;358:1336-45.

18 Wang S, Yiu KH, Mok MY, et al. Prevalence and extent of calcification over aorta, coronary and carotid arteries in patients with rheumatoid arthritis. J Intern Med 2009:266:445-52.

19 Yiu KH, Mok MY, Wang S, et al. Prognostic role of coronary calcification in patients with rheumatoid arthritis and systemic lupus erythematosus. Clin Exp Rheumatol 2012;30:345-50.

20 Arnett FC, Edworthy SM, Bloch DA, et al. The American Rheumatism Association 1987 revised criteria for the classification of rheumatoid arthritis. Arthritis Rheum 1988;31:315-24

21 Aletaha D, Neogi T, Silman AJ, et al. 2010 rheumatoid arthritis classification criteria: an American College of Rheumatology/European League Against Rheumatism collaborative initiative. Ann Rheum Dis 2010:69:1580-8.

22 Aletaha D, Neogi T, Silman AJ, et al. 2010 Rheumatoid arthritis classification criteria: an American College of Rheumatology/European League Against Rheumatism collaborative initiative. Arthritis Rheum 2010;62:2569-81.

23 European Association for Cardiovascular Prevention \& RehabilitationReiner $Z$ Catapano AL, De Backer G, Graham I, Taskinen MR, Wiklund O, et al. ESC/EAS Guidelines for the management of dyslipidaemias: the Task Force for the management of dyslipidaemias of the European Society of Cardiology (ESC) and the European Atherosclerosis Society (EAS). Addenda. Addendum I. European Heart Journal 2011;32:1769-818. http://www.escardio.org/guidelines

24 Conroy RM, Pyörälä K, Fitzgerald AP, et al. Estimation of ten-year risk of fatal cardiovascular disease in Europe: the SCORE project. Eur Heart $J$ 2003;24:987-1003

25 Oudkerk M, Stillman AE, Halliburton SS, et al. Coronary artery calcium screening: current status and recommendations from the European Society of Cardiac
Radiology and North American Society for Cardiovascular Imaging. Eur Radio 2008; 18:2785-807.

26 Agatston AS, Janowitz WR. Quantification of coronary artery calcium using ultrafast computed tomography. J Am Coll Cardiol 1990;15:827-32.

27 Touboul PJ, Hennerici MG, Meairs S, et al. Mannheim carotid intima-media thickness consensus (2004-2006). An update on behalf of the Advisory Board of the 3rd and 4th Watching the Risk Symposium, 13th and 15th European Stroke Conferences, Mannheim, Germany, 2004, and Brussels, Belgium, 2006. Cerebrovasc Dis 2007;23:75-80.

28 Folsom AR, Kronmal RA, Detrano RC, et al. Coronary artery calcification compared with carotid intima-media thickness in the prediction of cardiovascular disease incidence: the Multi-Ethnic Study of Atherosclerosis (MESA). Arch Intern Med 2008;168:1333-9.

29 Newman AB, Naydeck BL, Ives DG, et al. Coronary artery calcium, carotid artery wall thickness, and cardiovascular disease outcomes in adults 70 to 99 years old. Am J Cardiol 2008;101:186-92.

30 Ikeda N, Kogame N, lijima R, et al. Carotid artery intima-media thickness and plaque score can predict the SYNTAX score. Eur Heart $J$ 2012:33:113-19.

31 Spence JD. Ultrasound measurement of carotid plaque as a surrogate outcome for coronary artery disease. Am J Cardiol 2002;89:10B-5B; discussion 15B-16B.

32 Lester SJ, Eleid MF, Khandheria BK, et al. Carotid intima-media thickness and coronary artery calcium score as indications of subclinical atherosclerosis. Mayo Clin Proc 2009;84:229-33.

33 Naqvi TZ, Mendoza F, Rafii F, et al. High prevalence of ultrasound detected carotid atherosclerosis in subjects with low Framingham risk score: potential implications for screening for subclinical atherosclerosis. Am Soc Echocardiogr 2010;23:809-15. 